\title{
Preservation of Piecewise Constancy under TV Regularization with Rectilinear Anisotropy*
}

\author{
Clemens Kirisits $^{1}$, Eric Setterqvist ${ }^{1}$, and Otmar Scherzer ${ }^{1,2}$ \\ ${ }^{1}$ Faculty of Mathematics, University of Vienna, Vienna, Austria \\ ${ }^{2}$ Johann Radon Institute for Computational and Applied Mathematics (RICAM), \\ Austrian Academy of Sciences, Linz, Austria
}

March 11, 2019

\begin{abstract}
A recent result by Łasica, Moll and Mucha about the $\ell^{1}$-anisotropic RudinOsher-Fatemi model in $\mathbb{R}^{2}$ asserts that the solution is piecewise constant on a rectilinear grid, if the datum is. By means of a new proof we extend this result to $\mathbb{R}^{n}$. The core of our proof consists in showing that averaging operators associated to certain rectilinear grids map subgradients of the $\ell^{1}$-anisotropic total variation seminorm to subgradients.
\end{abstract}

\section{Introduction}

This article is concerned with a variant of the Rudin-Osher-Fatemi (ROF) image denoising model [13]. More specifically, we consider minimization of

$$
\frac{1}{2}\|u-f\|_{L^{2}}^{2}+\alpha J(u)
$$

where $J(u)=\int_{\Omega}\|\nabla u(x)\|_{\ell^{1}} d x$ is the total variation with $\ell^{1}$-anisotropy. This model and variations thereof have been used in imaging applications for data exhibiting a rectilinear geometry [2, 7, 14, 15. Numerical algorithms for minimizing (1) have been studied, for example, in [6, 10, 12].

The $\ell^{1}$-anisotropic total variation has a special property from a theoretical point of view as well. It has been shown in 3, Thm. 3.4, Rem. 3.5] that approximation of a general $u \in B V \cap L^{p}$ by functions $u_{m}$ piecewise constant on rectilinear grids, in the sense that

$$
\left\|u-u_{m}\right\|_{L^{p}} \rightarrow 0 \quad \text { and } \quad J\left(u_{m}\right) \rightarrow J(u),
$$

is not possible for $J(u)=\int_{\Omega}\|\nabla u(x)\|_{\ell^{q}} d x$, unless $q=1$.

Let $\Omega \subset \mathbb{R}^{n}$ be a finite union of hyperrectangles, each aligned with the coordinate axes. Our main result, Theorem 3.6] states that if the given function $f: \Omega \rightarrow \mathbb{R}$ is piecewise constant on a rectilinear grid, then the minimizer of (1) is too. This extends a recent result by Easica, Moll and Mucha about two-dimensional domains [11. Thm. 5]. Their proof is based on constructing the solution by means of its level sets and relies on minimization of an anisotropic Cheeger-type functional over subsets of $\Omega$.

*The final authenticated publication is available online at https ://doi.org/10.1007/978-3-030-22368-7_40 
The proof we present below is centred around the averaging operator $A_{G}$ associated to the grid $G$ on which $f$ is piecewise constant. In addition to being a contraction, it has the crucial property of mapping subgradients of $J$ to subgradients, that is, $A_{G}(\partial J(0)) \subset \partial J(0)$, see Theorem 3.2. Combined with the dual formulation of (1) we obtain that the minimizer must be piecewise constant on the same grid as $f$. While it might be possible to extend the techniques of [11] to higher dimensions, we believe that modifying the so-called "squaring step" in the proof of [11, Lem. 2] could lead to difficulties.

Theorem 3.6 implies that, if $f$ is piecewise constant on a rectilinear grid, then minimization of functional (1) becomes a finite-dimensional problem. More precisely, in this case the solution can be found by minimizing a discrete energy of the form

$$
\sum_{i} w_{i}\left|u_{i}-f_{i}\right|^{2}+\alpha \sum_{i, j} w_{i j}\left|u_{i}-u_{j}\right|
$$

where the weights $w_{i}, w_{i j} \geq 0$ depend only on the grid. For problems of this sort there are many efficient algorithms, such as graph cuts [4, 5, 8]. Extending the preservation of piecewise constancy to domains $\Omega \subset \mathbb{R}^{n}, n \geq 3$, means that the discrete reformulation can also be exploited for processing higher dimensional data such as volumetric images or videos.

This article is organized as follows. Section 2 contains the basic concepts that will be required throughout. In Section 2.1 we define several spaces of piecewise constant functions, while Section 2.2 is devoted to the $\ell^{1}$-anisotropic ROF model. Section 3 is the main part of this paper. It starts with introducing the averaging operator $A_{G}$ and ends with Theorem 3.6. The article is concluded in Section 4.

\section{Mathematical preliminaries}

\subsection{PCR functions}

In this section we introduce several notions related to functions which are piecewise constant on rectilinear subsets of $\mathbb{R}^{n}$. Some of these are $n$-dimensional analogues of notions from [11, Sec. 2.3].

A bounded set $R \subset \mathbb{R}^{n}$ which can be written as a Cartesian product of $n$ proper intervals is called an $n$-dimensional hyperrectangle. Recall that an interval is proper, if it is neither empty nor a singleton. Finite unions of $n$-dimensional hyperrectangles will be referred to as rectilinear $n$-polytopes.

A rectilinear grid, or simply grid, is a finite family of affine hyperplanes, each being perpendicular to one of the coordinate axes of $\mathbb{R}^{n}$. For a rectilinear $n$-polytope $P$ we denote by $G(P)$ the smallest grid with the property that the union of all its affine hyperplanes contains the entire boundary of $P$.

Throughout this article $\Omega \subset \mathbb{R}^{n}$ is an open rectilinear $n$-polytope. A finite family of rectilinear $n$-polytopes $\mathcal{Q}=\left\{P_{1}, \ldots, P_{N}\right\}$ is called a partition of $\Omega$, if they have pairwise disjoint interiors and the union of their closures equals $\bar{\Omega}$. Every grid $G$ defines a partition $\mathcal{Q}(G)$ of $\Omega$ into rectilinear $n$-polytopes in the following way: $P \subset \Omega$ belongs to $\mathcal{Q}(G)$, if and only if its boundary is contained in $\partial \Omega \cup \cup G$ while int $P$ and $\bigcup G$ are disjoint. Note that if $G$ contains $G(\Omega)$, then $\mathcal{Q}(G)$ consists only of hyperrectangles.

We adopt the notation $P C R(\Omega)$, or simply $P C R$, from [11 for the set of all integrable functions $f: \Omega \rightarrow \mathbb{R}$ which can be written as finite linear combinations of indicator functions of rectilinear $n$-polytopes. That is, $f \in P C R$ if there is an 

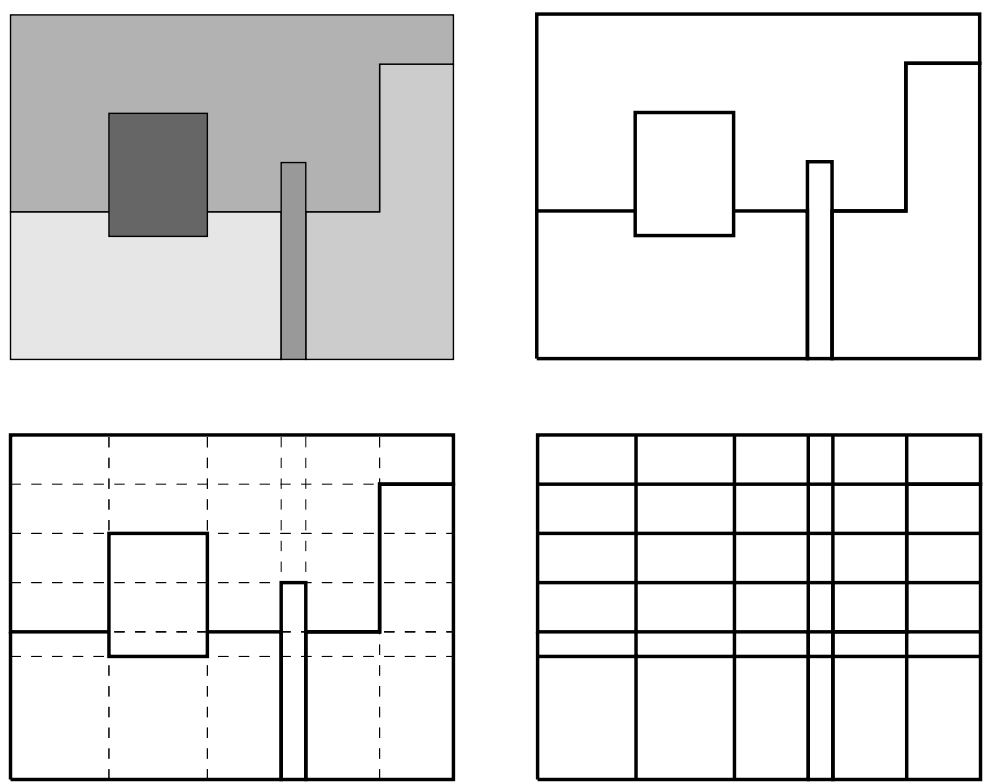

Figure 1: Upper left: A PCR function $f$ on a planar domain $\Omega$. Each level set of $f$ is visualized using a different grey tone. Upper right: The boundaries of the level sets of $f$. Lower left: Extension of the boundaries of the level sets of $f$. Lower right: The minimal grid $G_{f}$ (lines) and the partition $\mathcal{Q}\left(G_{f}\right)$ of $\Omega$ (rectangular cells).

$N \in \mathbb{N}, c_{i} \in \mathbb{R}$ and rectilinear $n$-polytopes $P_{i} \subset \Omega, 1 \leq i \leq N$, such that

$$
f=\sum_{i=1}^{N} c_{i} \mathbf{1}_{P_{i}}
$$

almost everywhere. Here, $\mathbf{1}_{A}$ is the indicator function of the set $A$, defined by

$$
\mathbf{1}_{A}(x)= \begin{cases}1, & x \in A, \\ 0, & x \notin A .\end{cases}
$$

We can assume, without loss of generality, that the values $c_{i}$ are pairwise distinct and that the polytopes $P_{i}$ are pairwise disjoint, which makes the representation (2) unique almost everywhere. To every $f \in P C R$ we associate its minimal grid, that is, the unique smallest grid covering the boundaries of all level sets of $f$, given by

$$
G_{f}=\bigcup_{i=1}^{N} G\left(P_{i}\right) .
$$

Note that the partition $\mathcal{Q}\left(G_{f}\right)$ always consists of hyperrectangles only. See Figure 1 for an illustration of $G_{f}$ and $\mathcal{Q}\left(G_{f}\right)$.

For a given grid $G$ we denote by $P C R_{G}$ the set of all functions in $P C R$ which are equal almost everywhere to a finite linear combination of indicator functions of $P_{i} \in \mathcal{Q}(G)$.

The following notions are essential for the proof of Theorem 3.2 . Fix an $i \in$ $\{1, \ldots, n\}$ as well as coordinates $x_{1}, \ldots, x_{i-1}, x_{i+1}, \ldots, x_{n}$. The set $\left\{x_{i} \in \mathbb{R}\right.$ : $\left.\left(x_{1}, \ldots, x_{n}\right) \in \Omega\right\}$ is a union of finitely many disjoint intervals

$$
I_{i}^{k}=\left(a_{i}^{k}, b_{i}^{k}\right), \quad k=1, \ldots, m .
$$


Note that the number of intervals $m$ as well as the intervals $I_{i}^{k}$ themselves depend on, and are uniquely determined by, the coordinates $x_{1}, \ldots, x_{i-1}, x_{i+1}, \ldots, x_{n}$. For an illustration of the intervals $I_{i}^{k}$ see Example 2.1 below. Next, let $\mathcal{G}$ be the set of all rectilinear grids of $\mathbb{R}^{n}$. For every $G \in \mathcal{G}$ and $i \in\{1, \ldots, n\}$ we define

$$
\Gamma_{G}^{i}=\left\{g \in P C R_{G}: \sup _{s \in\left(a_{i}^{k}, b_{i}^{k}\right)}\left|\int_{a_{i}^{k}}^{s} g d x_{i}\right| \leq 1, \int_{a_{i}^{k}}^{b_{i}^{k}} g d x_{i}=0,1 \leq k \leq m\right\} .
$$

The restrictions on $g$ are to be understood for almost every

$$
\left(x_{1}, \ldots, x_{i-1}, x_{i+1}, \ldots, x_{n}\right) \in \mathbb{R}^{n-1}
$$

such that there is an $x_{i} \in \mathbb{R}$ satisfying $\left(x_{1}, \ldots, x_{n}\right) \in \Omega$. The sum of the spaces $\Gamma_{G}^{i}$ is denoted by

$$
\Gamma_{G}=\left\{\sum_{i=1}^{n} g_{i}: g_{i} \in \Gamma_{G}^{i}, 1 \leq i \leq n\right\}
$$

and we further set

$$
\Gamma=\bigcup_{G \in \mathcal{G}} \Gamma_{G} .
$$

Remark 2.1. The set $\Gamma_{G}$ consists of divergences of certain piecewise affine vector fields. More precisely, Theorem 3.2 below implies that $\Gamma_{G}=\partial J(0) \cap P C R_{G}$, that is, $\Gamma_{G}$ is the set of all subgradients of $J$ which are piecewise constant on $G$.

Finally, those elements of $\Gamma_{G}^{i}$ which have compact support in $\Omega$ are collected in the set $\Gamma_{G, c}^{i}$, and we define analogously

$$
\begin{aligned}
\Gamma_{G, c} & =\left\{\sum_{i=1}^{n} g_{i}: g_{i} \in \Gamma_{G, c}^{i}, 1 \leq i \leq n\right\}, \\
\Gamma_{c} & =\bigcup_{G \in \mathcal{G}} \Gamma_{G, c} .
\end{aligned}
$$

Example 2.1. For the rectilinear 2-polytope $\Omega$ of Figure $Q$ we have the following intervals $I_{1}^{k}$ and $I_{2}^{k}$

$$
\left\{x_{1} \in \mathbb{R}:\left(x_{1}, x_{2}\right) \in \Omega\right\}= \begin{cases}(0,6), & \text { if } x_{2} \in(0,1) \cup(2,3), \\ (0,2) \cup(4,6), & \text { if } x_{2} \in[1,2], \\ (3,6), & \text { if } x_{2} \in[3,4),\end{cases}
$$

and

$$
\left\{x_{2} \in \mathbb{R}:\left(x_{1}, x_{2}\right) \in \Omega\right\}= \begin{cases}(0,3), & \text { if } x_{1} \in(0,2), \\ (0,1) \cup(2,3), & \text { if } x_{1} \in[2,3] \\ (0,1) \cup(2,4), & \text { if } x_{1} \in(3,4] \\ (0,4), & \text { if } x_{1} \in(4,6) .\end{cases}
$$

\subsection{The $\ell^{1}$-anisotropic ROF model}

The notion of anisotropic total variation was introduced in [1. In this article we exclusively consider one particular variant. 


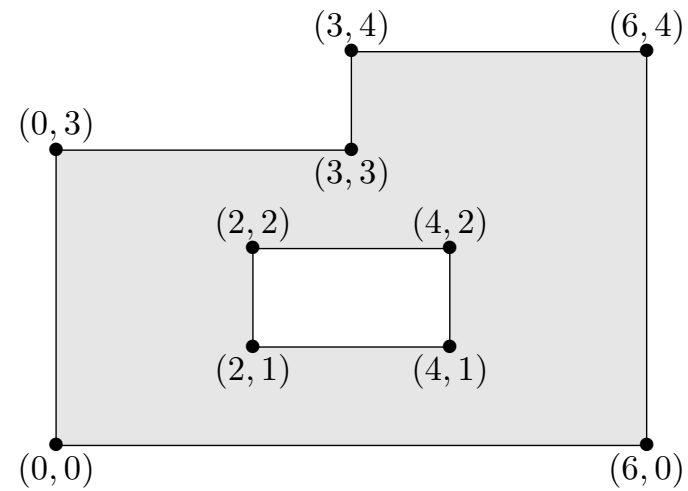

Figure 2: A rectilinear 2-polytope $\Omega$.

For every $\alpha>0$ we denote by $\mathcal{B}_{\alpha}$ the set of all smooth compactly supported vector fields on $\Omega$ whose components are bounded by $\alpha$, that is,

$$
\mathcal{B}_{\alpha}=\left\{H \in C_{c}^{\infty}\left(\Omega, \mathbb{R}^{n}\right): \max _{1 \leq i \leq n}\left|H_{i}(x)\right| \leq \alpha, \forall x \in \Omega\right\} .
$$

The $\ell^{1}$-anisotropic total variation $J: L^{2}(\Omega) \rightarrow \mathbb{R} \cup\{+\infty\}$ is given by

$$
J(u)=\sup _{H \in \mathcal{B}_{1}} \int_{\Omega} u \operatorname{div} H d x=\sup _{h \in \overline{\operatorname{div} \mathcal{B}_{1}}} \int_{\Omega} u h d x,
$$

where the bar denotes closure in $L^{2}(\Omega)$. Thus, $J$ is the support function of the closed and convex set $\overline{\operatorname{div} \mathcal{B}_{1}}$, which implies that $\overline{\operatorname{div} \mathcal{B}_{1}}=\partial J(0)$, or more generally

$$
\overline{\operatorname{div} \mathcal{B}_{\alpha}}=\alpha \partial J(0)
$$

for every $\alpha>0$. If $u$ is a Sobolev function, then $J(u)=\int_{\Omega}\|\nabla u(x)\|_{\ell^{1}} d x$.

The next lemma states that the $\ell^{1}$-anisotropic ROF model is equivalent to constrained $L^{2}$-minimization. However, the way it is formulated it actually applies to every support function $J$ of a closed and convex subset of $L^{2}(\Omega)$.

Lemma 2.1. For every $\alpha>0$ and $f \in L^{2}(\Omega)$ the minimization problem

$$
\min _{u \in L^{2}(\Omega)} \frac{1}{2}\|u-f\|_{L^{2}}^{2}+\alpha J(u)
$$

is equivalent to

$$
\min _{u \in f-\alpha \partial J(0)}\|u\|_{L^{2}}
$$

Proof. The dual problem associated to (6) is given by

$$
\min _{w \in L^{2}(\Omega)} \frac{1}{2}\|w-f\|_{L^{2}}^{2}+(\alpha J)^{*}(w)
$$

where the asterisk stands for convex conjugation. The two solutions $u_{\alpha}$ and $w_{\alpha}$ of (6) and (7), respectively, satisfy the optimality conditions

$$
\begin{gathered}
u_{\alpha}=f-w_{\alpha}, \\
w_{\alpha} \in \partial(\alpha J)\left(u_{\alpha}\right) .
\end{gathered}
$$


Concerning the derivation of (7) and (8) we refer to [9, Chap. III, Rem. 4.2]. Since $\alpha J$ is the support function of the set $\alpha \partial J(0)$, recall (4), its conjugate is the characteristic function

$$
(\alpha J)^{*}(w)= \begin{cases}0, & w \in \alpha \partial J(0), \\ +\infty, & w \notin \alpha \partial J(0) .\end{cases}
$$

Therefore, problem (7) is equivalent to

$$
\min _{w \in \alpha \partial J(0)}\|w-f\|_{L^{2}} .
$$

Finally, using the optimality condition (8) we get

$$
\left\|u_{\alpha}\right\|_{L^{2}}=\left\|w_{\alpha}-f\right\|_{L^{2}}=\min _{w \in \alpha \partial J(0)}\|w-f\|_{L^{2}}=\min _{u \in f-\alpha \partial J(0)}\|u\|_{L^{2}} .
$$

\section{The averaging operator $A_{G}$}

Let $\Omega$ be a rectilinear $n$-polytope and $G$ a grid. Define the averaging operator $A_{G}: L^{1}(\Omega) \rightarrow P C R_{G}(\Omega)$ by

$$
A_{G} g=\sum_{i=1}^{N}\left(\frac{1}{\left|P_{i}\right|} \int_{P_{i}} g(s) d s\right) \mathbf{1}_{P_{i}},
$$

where $P_{i} \in \mathcal{Q}(G)$ and $\left|P_{i}\right|$ is its $n$-dimensional volume.

Two properties of the operator $A_{G}$ turn out to be important when establishing the main result of this paper, Theorem 3.6. The first one is

Lemma 3.1. For every $u \in L^{1}(\Omega)$ and convex $\varphi: \mathbb{R} \rightarrow \mathbb{R}$

$$
\int_{\Omega} \varphi\left(\left(A_{G} u\right)(x)\right) d x \leq \int_{\Omega} \varphi(u(x)) d x .
$$

Proof. By applying Jensen's inequality we obtain

$$
\begin{aligned}
\int_{\Omega} \varphi\left(\left(A_{G} u\right)(x)\right) d x & =\sum_{i=1}^{N} \int_{P_{i}} \varphi\left(\left(A_{G} u\right)(x)\right) d x=\sum_{i=1}^{N} \varphi\left(\frac{1}{\left|P_{i}\right|} \int_{P_{i}} u(x) d x\right)\left|P_{i}\right| \\
& \leq \sum_{i=1}^{N} \int_{P_{i}} \varphi(u(x)) d x=\int_{\Omega} \varphi(u(x)) d x .
\end{aligned}
$$

Remark 3.1. Lemma 3.1 implies in particular that $A_{G}$ is a contraction,

$$
\left\|A_{G}\right\|_{L^{p} \rightarrow L^{p}} \leq 1, \quad 1 \leq p<\infty .
$$

The second property of $A_{G}$ is that it maps subgradients of $J$ to subgradients. Recall that $G(\Omega)$ is the smallest grid covering the entire boundary of $\Omega$.

Theorem 3.2. Let $G$ be a grid containing $G(\Omega)$. Then $A_{G}(\partial J(0)) \subset \partial J(0)$.

Proof. The proof is divided into three steps, each being proved in a separate lemma

$$
A_{G}(\partial J(0)) \stackrel{\text { Lem. } 3.3}{\subset} \Gamma_{G} \subset \Gamma \stackrel{\text { Lem. } \subset .4}{\subset} \bar{\Gamma}_{c} \stackrel{\text { Lem. } 3.5}{\subset} \partial J(0) .
$$

Note that the inclusion $\Gamma_{G} \subset \Gamma$ is trivial. 
Lemma 3.3. Let $G$ be a grid containing $G(\Omega)$. Then $A_{G}(\partial J(0)) \subset \Gamma_{G}$.

Proof. Throughout this proof we exploit the fact that $\partial J(0)=\overline{\operatorname{div} \mathcal{B}_{1}}$, recall equation (5).

First, note that $P C R_{G}$ is a finite-dimensional subspace of $L^{2}$ and that the sets $\Gamma_{G}^{i}, i=1, \ldots, n$, are bounded and closed subsets of $P C R_{G}$. It follows that $\Gamma_{G}$ is a closed subset of $P C R_{G}$ and in particular of $L^{2}$. Therefore, it suffices to show $A_{G}\left(\operatorname{div} \mathcal{B}_{1}\right) \subset \Gamma_{G}$, as we then have $A_{G}\left(\overline{\operatorname{div} \mathcal{B}_{1}}\right) \subset \overline{A_{G}\left(\operatorname{div} \mathcal{B}_{1}\right)} \subset \overline{\Gamma_{G}}=\Gamma_{G}$, because $A_{G}$ is continuous.

Take $H=\left(H_{1}, \ldots, H_{n}\right) \in \mathcal{B}_{1}$. We want to show that $A_{G} \partial H_{i} / \partial x_{i} \in \Gamma_{G}^{i}$, that is,

$$
\sup _{s \in\left(a_{i}^{k}, b_{i}^{k}\right)}\left|\int_{a_{i}^{k}}^{s} A_{G} \frac{\partial H_{i}}{\partial x_{i}} d x_{i}\right| \leq 1, \quad \text { and } \quad \int_{a_{i}^{k}}^{b_{i}^{k}} A_{G} \frac{\partial H_{i}}{\partial x_{i}} d x_{i}=0
$$

for $i=1, \ldots, n$ and each $k$, where $\left(a_{i}^{k}, b_{i}^{k}\right)$ are the intervals defined in equation (3).

Consider the second integral first. From the definition of $A_{G}$ it follows that $\left(a_{i}^{k}, b_{i}^{k}\right)$ can be divided into a finite number of subintervals in such a way that the integrand is constant on each. In addition the assumption $G \supset G(\Omega)$ implies that the partition $\mathcal{Q}(G)$ consists of hyperrectangles only. Thus, after a potential relabelling of the $R_{j} \in \mathcal{Q}(G)$, we can write

$$
\begin{aligned}
\int_{a_{i}^{k}}^{b_{i}^{k}} A_{G} \frac{\partial H_{i}}{\partial x_{i}} d x_{i} & =\sum_{j=1}^{M} \int_{s_{j-1}}^{s_{j}}\left(\frac{1}{\left|R_{j}\right|} \int_{R_{j}} \frac{\partial H_{i}}{\partial x_{i}} d x\right) d x_{i} \\
& =\sum_{j=1}^{M} \frac{s_{j}-s_{j-1}}{\left|R_{j}\right|} \int_{R_{j}} \frac{\partial H_{i}}{\partial x_{i}} d x
\end{aligned}
$$

for some $M \in\{1, \ldots, N\}$ and $a_{i}^{k}=s_{0}<s_{1}<\cdots<s_{M}=b_{i}^{k}$. Note that $\left|R_{j}\right| /\left(s_{j}-\right.$ $\left.s_{j-1}\right)$ is the $(n-1)$-dimensional volume of $\partial R_{j} \cap \partial R_{j+1}$, and that this volume is independent of $j \in\{1, \ldots M\}$. In other words, the hyperrectangles $R_{j}$ only differ in their extent in $x_{i}$-direction, compare Figure 1, bottom right. The reason is that $\mathcal{Q}(G)$ is not an arbitrary partition of $\Omega$ into hyperrectangles, but rather formed by a grid. Setting $C=\left(s_{j}-s_{j-1}\right) /\left|R_{j}\right|$ we further obtain

$$
=C \int_{\bigcup_{j} R_{j}} \frac{\partial H_{i}}{\partial x_{i}} d x
$$

The remaining integral can be computed by turning it into an iterated one, integrating with respect to $x_{i}$ first, and recalling that $H$ is compactly supported

$$
=C \underbrace{\int \cdots \int}_{n-1} \int_{a_{i}^{k}}^{b_{i}^{k}} \frac{\partial H_{i}}{\partial x_{i}} d x_{i}=\left.C \int \cdots \int H_{i}\right|_{x_{i}=a_{i}^{k}} ^{x_{i}=b_{i}^{k}}=0 .
$$

Here $\left.F\right|_{x_{i}=a} ^{x_{i}=b}$ stands for $F\left(x_{i}=b\right)-F\left(x_{i}=a\right)$, where $F\left(x_{i}=c\right)$ denotes the restriction of $F$ to the affine hyperplane defined by $x_{i}=c$.

Now integrate up to an arbitrary $s \in\left(a_{i}^{k}, b_{i}^{k}\right]$. We can assume $s \in\left(s_{\ell-1}, s_{\ell}\right]$ for some $\ell \in\{1, \ldots, M\}$ and a brief computation similar to the one above shows that

$$
\begin{aligned}
\int_{a_{i}^{k}}^{s} A_{G} \frac{\partial H_{i}}{\partial x_{i}} d x_{i} & =\left.C \int \cdots \int H_{i}\right|_{x_{i}=a_{i}^{k}} ^{x_{i}=s_{\ell-1}}+\left.\frac{s-s_{\ell-1}}{\left|R_{\ell}\right|} \int \cdots \int H_{i}\right|_{x_{i}=s_{\ell-1}} ^{x_{i}=s} \\
& =C \int \cdots \int H_{i}\left(x_{i}=s_{\ell-1}\right)+\left.\frac{s-s_{\ell-1}}{\left|R_{\ell}\right|} \int \cdots \int H_{i}\right|_{x_{i}=s_{\ell-1}} ^{x_{i}=s} .
\end{aligned}
$$




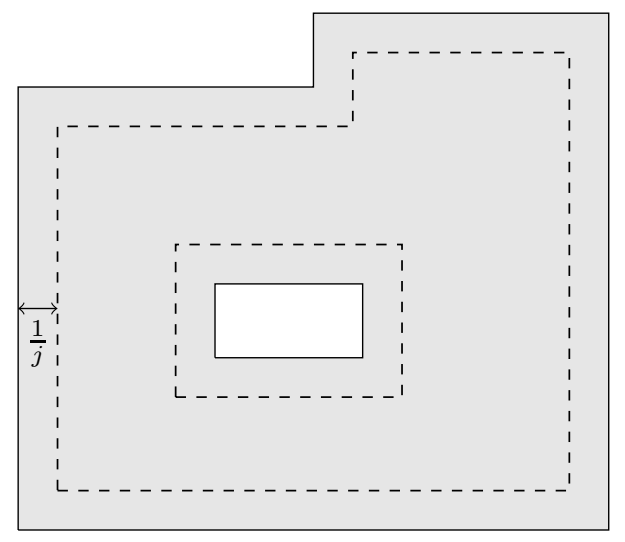

Figure 3: The construction of $\Omega_{j}$ by removing strips of width $1 / j$ from $\Omega$.
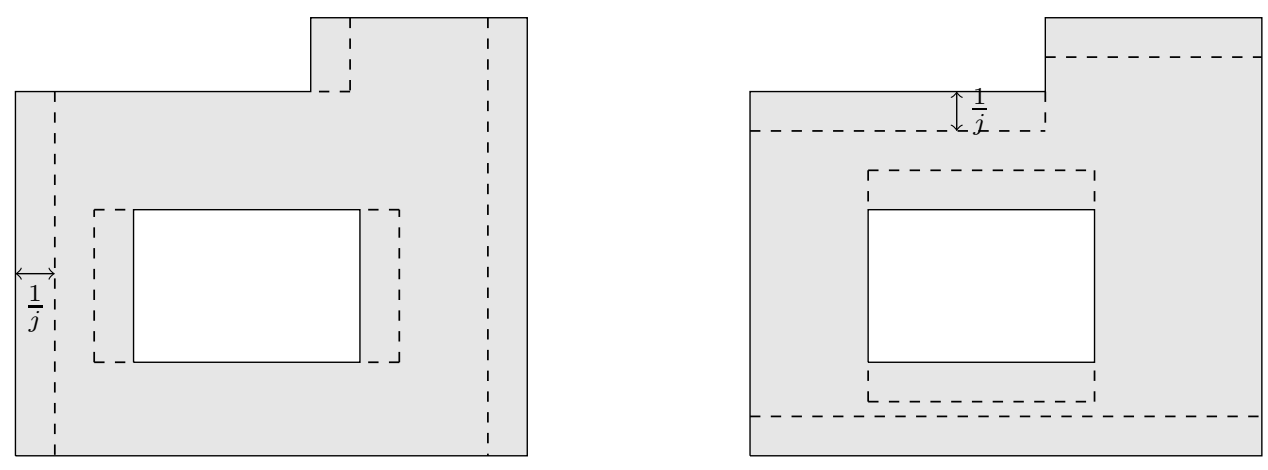

Figure 4: The construction of $\Omega_{j}^{1}$ (left) and $\Omega_{j}^{2}$ (right) by removing strips from $\Omega_{j}$.

Recalling that we can write $C=\left(s_{\ell}-s_{\ell-1}\right) /\left|R_{\ell}\right|$ we rearrange terms

$$
=\frac{s_{\ell}-s}{\left|R_{\ell}\right|} \int \cdots \int H_{i}\left(x_{i}=s_{\ell-1}\right)+\frac{s-s_{\ell-1}}{\left|R_{\ell}\right|} \int \cdots \int H_{i}\left(x_{i}=s\right) .
$$

Finally, we estimate $H_{i} \leq 1$ and obtain

$$
\leq \frac{s_{\ell}-s}{\left|R_{\ell}\right|} \frac{\left|R_{\ell}\right|}{s_{\ell}-s_{\ell-1}}+\frac{s-s_{\ell-1}}{\left|R_{\ell}\right|} \frac{\left|R_{\ell}\right|}{s_{\ell}-s_{\ell-1}}=1
$$

Similarly, we get $\int_{a_{i}^{k}}^{s} A_{G} \frac{\partial H_{i}}{\partial x_{i}} d x_{i} \geq-1$. Thus we have $A_{G} \partial H_{i} / \partial x_{i} \in \Gamma_{G}^{i}$.

Lemma 3.4. $\Gamma \subset \overline{\Gamma_{c}}$.

Proof. Let $j \in \mathbb{N}$ and define $\Omega_{j} \subset \Omega$ by removing strips of width $1 / j$ from the boundary of $\Omega$. It is assumed that $j$ is chosen large enough such that the strips are contained in $\Omega$. See Figure 3 for an example of the construction of $\Omega_{j}$ in the plane. Next, for $i=1, \ldots, n$ we define $\Omega_{j}^{i} \subset \Omega_{j}$, by removing strips of width $1 / j$ from those parts of the boundary of $\Omega_{j}$ which are orthogonal to the $x_{i}$-axis. By choosing $j$ large enough, the strips will be contained in $\Omega_{j}$. For an illustration of the construction of $\Omega_{j}^{i}$ in the plane, see Figure 4 .

Take $h \in \Gamma$. So, $h \in \Gamma_{G}$ for some $G \in \mathcal{G}$ and in particular $h=\sum_{i=1}^{n} h_{i}$ where 
$h_{i} \in \Gamma_{G}^{i}$. Let $g_{j}=\sum_{i=1}^{n} g_{j, i}$ where

$$
g_{j, i}(x)= \begin{cases}0, & \text { if } x \in \Omega \backslash \Omega_{j}, \\ 2 h_{i}(x), & \text { if } x \in \Omega_{j} \backslash \Omega_{j}^{i}, \\ h_{i}(x), & \text { otherwise. }\end{cases}
$$

Note that there is a grid $G_{j} \supset G$ such that $g_{j, i} \in P C R_{G_{j}}$ for every $i=1, \ldots, n$, and that for $j$ large enough

$$
\left|\int_{a_{i}^{k}}^{s} g_{j, i} d x_{i}\right| \leq\left|\int_{a_{i}^{k}}^{s} h_{i} d x_{i}\right|
$$

for every interval $\left(a_{i}^{k}, b_{i}^{k}\right)$, recall equation (3), and $s \in\left(a_{i}^{k}, b_{i}^{k}\right]$. It follows that $g_{j, i} \in \Gamma_{G_{j}, c}^{i}$ and therefore $g_{j} \in \Gamma_{G_{j}, c}$. Finally, it can be directly verified that

$$
\lim _{j \rightarrow \infty}\left\|g_{j}-h\right\|_{L^{2}}=0 .
$$

As $h \in \Gamma$ was chosen arbitrarily we conclude that $\Gamma \subset \overline{\Gamma_{c}}$.

Lemma 3.5. $\overline{\Gamma_{c}} \subset \partial J(0)$.

Proof. As in Lemma 3.3 we use the fact that $\partial J(0)=\overline{\operatorname{div} \mathcal{B}_{1}}$.

Take $h \in \Gamma_{c}$. So there is a grid $G$ such that $h=\sum_{i=1}^{n} h_{i} \in \Gamma_{G, c}$ where $h_{i} \in \Gamma_{G, c}^{i}$. From $h$ we now construct a vector field $H=\left(H_{1}, \ldots, H_{n}\right)$. For every $i \in\{1, \ldots, n\}$ and $x \in \Omega$ there is a unique interval $I_{i}^{k}=\left(a_{i}^{k}, b_{i}^{k}\right)$ containing $x_{i}$, recall equation (3). Based on this observation we define the components of $H$ by

$$
H_{i}(x)=\int_{a_{i}^{k}}^{x_{i}} h_{i}\left(x_{1}, \ldots, x_{i-1}, s, x_{i+1} \ldots, x_{n}\right) d s .
$$

It follows that $\left\|H_{i}\right\|_{L^{\infty}} \leq 1$ and $\operatorname{supp}\left(H_{i}\right) \subset \Omega$. $H$ is now modified into a vector field belonging to $\mathcal{B}_{1}$. Let $\left\{\rho_{j}\right\}_{j \in \mathbb{N}}$ denote a sequence of mollifiers on $\mathbb{R}^{n}$ supported on the closed Euclidean ball centred at 0 with radius $1 / j$. Recalling standard results regarding convolution and mollifiers, we derive $\left\|H_{i} * \rho_{j}\right\|_{L^{\infty}} \leq\left\|H_{i}\right\|_{L^{\infty}}\left\|\rho_{j}\right\|_{L^{1}}=$ $\left\|H_{i}\right\|_{L^{\infty}} \leq 1$ and moreover, for $j$ large enough, $H_{i} * \rho_{j} \in C_{c}^{\infty}(\Omega)$. Hence, for $j \in \mathbb{N}$ large enough, the modification $H_{\rho_{j}}$ of $H$ given by

$$
H_{\rho_{j}}=\left(H_{1} * \rho_{j}, \ldots, H_{n} * \rho_{j}\right)
$$

is in $\mathcal{B}_{1}$. It follows that $h \in \overline{\operatorname{div} \mathcal{B}_{1}}$, as

$$
\begin{aligned}
\left\|\operatorname{div} H_{\rho_{j}}-h\right\|_{L^{2}} & =\left\|\sum_{i=1}^{n}\left(\frac{\partial}{\partial x_{i}}\left(H_{i} * \rho_{j}\right)-h_{i}\right)\right\|_{L^{2}}=\left\|\sum_{i=1}^{n}\left(h_{i} * \rho_{j}-h_{i}\right)\right\|_{L^{2}} \\
& \leq \sum_{i=1}^{n}\left\|h_{i} * \rho_{j}-h_{i}\right\|_{L^{2}} \stackrel{j \rightarrow \infty}{\longrightarrow} 0 .
\end{aligned}
$$

The element $h \in \Gamma_{c}$ was chosen arbitrarily and $\overline{\operatorname{div} \mathcal{B}_{1}}$ is closed, therefore $\overline{\Gamma_{c}} \subset$ $\overline{\operatorname{div} \mathcal{B}_{1}}$

\subsection{Preservation of piecewise constancy}

We are now ready to prove the following result. 
Theorem 3.6. Given $f \in P C R$ with minimal grid $G_{f}$, the minimizer $u_{\alpha}$ of the corresponding anisotropic ROF functional

$$
\min _{u \in L^{2}(\Omega)} \frac{1}{2}\|u-f\|_{L^{2}}^{2}+\alpha J(u)
$$

lies in $P C R_{G_{f}}$.

Proof. Recall that, according to Lemma 2.1, $u_{\alpha}$ is the unique element with minimal $L^{2}$-norm in $f-\alpha \partial J(0)$. From Theorem 3.2 and the fact that $A_{G_{f}} f=f$ it follows that also $A_{G_{f}} u_{\alpha} \in f-\alpha \partial J(0)$. As $\left\|A_{G_{f}} u_{\alpha}\right\|_{L^{2}} \leq\left\|u_{\alpha}\right\|_{L^{2}}$, because of Remark 3.1, we have $A_{G_{f}} u_{\alpha}=u_{\alpha}$. Therefore, $u_{\alpha} \in P C R_{G_{f}}$.

\section{Conclusion}

In [11, Thm. 5] the authors have shown that, for $\Omega$ being a rectilinear 2-polytope, $f \in P C R$ implies $u_{\alpha} \in P C R$. We have extended this preservation of piecewise constancy to rectilinear $n$-polytopes. Our proof can be summarized in the following way

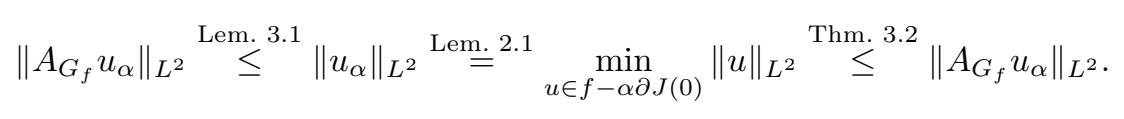

The crucial step is Theorem 3.2, asserting that

$$
A_{G}(\partial J(0)) \subset \partial J(0)
$$

which exploits the fact that the anisotropy of $J$ is compatible with the rectilinearity of the grid $G$.

\section{Acknowledgements}

We acknowledge support by the Austrian Science Fund (FWF) within the national research network "Geometry + Simulation," S117, subproject 4.

\section{References}

[1] M. Amar and G. Bellettini. A notion of total variation depending on a metric with discontinuous coefficients. Ann. Inst. H. Poincaré Anal. Non Linéaire, 11(1):91-133, 1994.

[2] B. Berkels, M. Burger, M. Droske, O. Nemitz, and M. Rumpf. Cartoon extraction based on anisotropic image classification. In Vision, Modeling, and Visualization Proceedings, pages 293-300, 2006.

[3] E. Casas, K. Kunisch, and C. Pola. Regularization by functions of bounded variation and applications to image enhancement. Appl. Math. Optim., 40(2):229-257, 1999.

[4] A. Chambolle. Total variation minimization and a class of binary MRF models. In A. Rangarajan, B. Vemuri, and A. L. Yuille, editors, Energy Minimization Methods in Computer Vision and Pattern Recognition, volume 3757 of Lecture Notes in Computer Vision, pages 136-152. Springer Berlin, Heidelberg, 2005.

[5] A. Chambolle and J. Darbon. On total variation minimization and surface evolution using parametric maximum flows. Int. J. Comput. Vision, 84(3):288307, April 2009. 
[6] H. Chen, C. Wang, Y. Song, and Z. Li. Split Bregmanized anisotropic total variation model for image deblurring. Journal of Visual Communication and Image Representation, 31:282-293, 2015.

[7] R. Choksi, Y. van Gennip, and A. Oberman. Anisotropic total variation regularized $L^{1}$ approximation and denoising/deblurring of $2 \mathrm{~d}$ bar codes. Inverse Problems \& Imaging, 5(3):591-617, 2011.

[8] J. Darbon and M. Sigelle. Image restoration with discrete constrained total variation. Part I: Fast and exact optimization. J. Math. Imaging Vision, 26(3):261-276, 2006.

[9] I. Ekeland and R. Temam. Convex Analysis and Variational Problems. NorthHolland, Amsterdam, 1976.

[10] T. Goldstein and S. Osher. The split Bregman method for L1-regularized problems. SIAM J. Imaging Sciences, 2:323-343, 2009.

[11] M. Łasica, S. Moll, and P. B. Mucha. Total variation denoising in $\ell^{1}$ anisotropy. SIAM J. Imaging Sciences, 10:1691-1723, 2017.

[12] Y. Li and F. Santosa. A computational algorithm for minimizing total variation in image restoration. IEEE Trans. Image Process., 5:987-995, 1996.

[13] L. Rudin, S. Osher, and E. Fatemi. Nonlinear total variation based noise removal algorithms. Physica D, 60:259-268, 1992.

[14] S. J. Sanabria, E. Ozkan, M. Rominger, and O. Goksel. Spatial domain reconstruction for imaging speed-of-sound with pulse-echo ultrasound: simulation and in vivo study. Phys. Med. Biol., 63(21):215015, October 2018.

[15] S. Setzer, G. Steidl, and T. Teuber. Restoration of images with rotated shapes. Numer. Algorithms, 48(1-3):49-66, 2008. 